\title{
Analysing the protection from respiratory tract infections and allergic diseases early in life by human milk components: the PRIMA birth cohort
}

\author{
Arthur H. van Stigt ${ }^{1}$, Katrien Oude Rengerink ${ }^{2}$, Kitty W. M. Bloemenkamp ${ }^{3}$, Wouter de Waal ${ }^{4}$, \\ Sabine M. P. J. Prevaes ${ }^{5}$, Thuy-My Le ${ }^{6}$, Femke van Wijk', Maaike Nederend ${ }^{1}$, Anneke H. Hellinga', \\ Christianne S. Lammers ${ }^{1}$, Gerco den Hartog ${ }^{7}$, Martijn J. C. van Herwijnen ${ }^{8}$, Johan Garssen ${ }^{9,10}$, \\ Léon M. J. Knippels ${ }^{9,10}$, Lilly M. Verhagen ${ }^{11}$, Caroline G. M. de Theije ${ }^{12}$, Alejandro Lopez-Rincon" ${ }^{9}$, \\ Jeanette H. W. Leusen' ${ }^{1}$, Belinda van't Land ${ }^{1,10}$ and Louis Bont ${ }^{1,11,13^{*}}$ PRIMA Initiative Group
}

\begin{abstract}
Background: Many studies support the protective effect of breastfeeding on respiratory tract infections. Although infant formulas have been developed to provide adequate nutritional solutions, many components in human milk contributing to the protection of newborns and aiding immune development still need to be identified. In this paper we present the methodology of the "Protecting against Respiratory tract Infections through human Milk Analysis" (PRIMA) cohort, which is an observational, prospective and multi-centre birth cohort aiming to identify novel functions of components in human milk that are protective against respiratory tract infections and allergic diseases early in life.

Methods: For the PRIMA human milk cohort we aim to recruit 1000 mother-child pairs in the first month postpartum. At one week, one, three, and six months after birth, fresh human milk samples will be collected and processed. In order to identify protective components, the level of pathogen specific antibodies, T cell composition, Human milk oligosaccharides, as well as extracellular vesicles (EVs) will be analysed, in the milk samples in relation to clinical data which are collected using two-weekly parental questionnaires. The primary outcome of this study is the number of parent-reported medically attended respiratory infections. Secondary outcomes that will be measured are physician diagnosed (respiratory) infections and allergies during the first year of life.

Discussion: The PRIMA human milk cohort will be a large prospective healthy birth cohort in which we will use an integrated, multidisciplinary approach to identify the longitudinal effect human milk components that play a role in preventing (respiratory) infections and allergies during the first year of life. Ultimately, we believe that this study will provide novel insights into immunomodulatory components in human milk. This may allow for optimizing formula feeding for all non-breastfed infants.
\end{abstract}

\footnotetext{
*Correspondence: L.Bont@umcutrecht.nl

${ }^{1}$ Center for Translational Immunology, University Medical Center Utrecht,

Utrecht, The Netherlands

Full list of author information is available at the end of the article
} original author(s) and the source, provide a link to the Creative Commons licence, and indicate if changes were made. The images or other third party material in this article are included in the article's Creative Commons licence, unless indicated otherwise in a credit line to the material. If material is not included in the article's Creative Commons licence and your intended use is not permitted by statutory regulation or exceeds the permitted use, you will need to obtain permission directly from the copyright holder. To view a copy of this licence, visit http://creativecommons.org/licenses/by/4.0/. The Creative Commons Public Domain Dedication waiver (http://creativeco mmons.org/publicdomain/zero/1.0/) applies to the data made available in this article, unless otherwise stated in a credit line to the data. 
Keywords: Breastfeeding, Human milk, Respiratory tract infections, Allergies, Immune development, Antibodies, Human milk oligosaccharides, T cells, Extracellular vesicles, Biobank

\section{Background}

\section{The burden of respiratory infections}

Respiratory infections are one of the major causes of illness in infants, specifically during the first 5 years of life [1]. It was estimated for 2015 that 703,900 children under the age of five died worldwide because of lower respiratory infections [2]. Most respiratory tract infections in children are caused by viral infections, with respiratory syncytial virus (RSV), influenza virus (IFV), parainfluenza (PIV) and metapneumovirus (MPV) being the most commonly found viruses [3, 4]. Each year, about 41.3-112.0 per 1000 children under one year of age were admitted to the hospital for RSV in Europe [5]. This leads to about 28,000 infants requiring medical care for RSV bronchiolitis in the Netherlands $[6,7]$ of which approximately 2000 require hospitalization. Hospitalisation for respiratory tract infections not only leads to considerable costs, but is also associated with recurrent wheeze, asthma and long term impaired lung function [8-11].

\section{Breastfeeding protects against infections}

Breastfeeding clearly has several benefits for infants, such as the capacity to protect against the risk and impact of neonatal infections $[12,13]$. For instance, infants that do not receive exclusive breastfeeding are at a higher risk of hospitalization in early life in relation to a wide range of common infections [14]. Exclusive breastfeeding until the age of four months followed by partial breastfeeding is associated with both reduction in gastrointestinal and respiratory infections $[15,16]$. Infants admitted to the hospital with RSV infection are less likely to have been breastfed [10, 17]. In addition, in a recent meta-analysis, one out of the eight risk factors for RSV-induced acute lower respiratory infection in children was "no breastfeeding", with an odds ratio of 2.24 (95\% CI 1.56-3.20) [18]. A similar protective effect of breastfeeding was observed for other common respiratory pathogens like influenza and invasive pneumococcal disease $[19,20]$.

A meta-analysis strongly favoured breastfeeding over formula feeding to reduce the risk of gastrointestinal infections (i.e. 0.36 ( $95 \%$ confidence interval (CI) 0.3 to 0.41)) [12]. This finding was supported by another metaanalysis that described a higher infant mortality due to diarrheal disease in children who were not exclusively breastfed the first six months of life [21].

The protective effect of breastfeeding on the development of allergic disease is less well pronounced compared to infectious diseases. In a meta-analysis it was found that breastfeeding protects from asthma at ages five to eighteen, eczema until the age of two and for allergic rhinitis until the age of five [22]. Similar results were seen in other studies, for instance the CHILD study that found that direct breastfeeding offers the best protection from asthma during the first three years of age $[23,24]$. However, there are also other studies demonstrating that there is even in increased risk on (mild) allergic diseases when children are being breastfed [25-27]. It is particularly hard to evaluate the effect of breastfeeding on the development of allergic diseases, as different definitions are used for allergic disease and different comparisons in duration of lactation [28]. Since the composition of breastfeeding is variable, it may well be that only some components in breastfeeding are protective against allergic diseases and not breastfeeding itself [28]. This hypothesis is supported by the findings of Lodge et al. where groups of human milk oligosaccharide (HMOs) were associated with an increased or reduced change of allergic diseases development in infants [29]. This suggests that breastfeeding contains protective components that may have immunomodulatory capacity.

\section{Protective components in breastfeeding}

Several components within human milk have been linked to the risk of infection during the first year of infancy. Primarily, antibodies in human milk are acknowledged to play a pivotal role protecting infants against various pathogens. Yet, it is still unclear which antibody repertoire would be favourable and what the effect of antibodies is compared to other human milk components. Breakey et al. demonstrated that high IgA antibody titres are linked to a reduction in infectious episodes whereas Mazur et al. described that RSV-specific maternal in breastfeeding IgG but not IgA antibodies are linked to reduction in the risk of RSV infections [30, 31]. Next to pathogen-specific antibodies human milk contains many more components with a protective capacity. For instance, Ramani et al. showed that higher concentrations of the HMO 2'-fucosyllactose (2'-FL) was associated with symptomatic rotaviral infections whereas in another study the presence of 2'-FL was associated with relative protection from respiratory infections [32, 33]. Addition of specific prebiotic oligosaccharide mixtures to infant formulas was shown to decrease the proportion of infections and allergic diseases in children when compared to the standard infant formula [34-38]. In vitro data suggests 
that HMOs exert their effects through various mechanisms. For instance, HMOs are known to influence interactions between immune cells (e.g. dendritic and $\mathrm{T}$ cells), stimulate mucosal barrier and immune system maturation, modulate immune responses following the formation of antigen-antibody complexes, and may affect the immune response following the formation of (endogenous) antibody-antigen complexes [39-44]. HMOs may also influence the gut microbiome of the infant. Breatsfed children usually have a gut microbiome dominated by Lactobacillus species and bifidobacteria compared to infant formula fed children [45, 46] Moreover, Ramani et al. fund that LNT (Lacto-Ntetraose) and 6'SL (6'-Sialyllactose) were both associated with Enterobacter and Klebsiella species [32]. In addition to pathogen-specific antibodies and HMOs, human milk contains many more components, including immune cells and extracellular vesicles (EVs) [4751]. Several miRNA detected in human milk EVs were immune-related miRNAs [52]. Activated and memory $\mathrm{T}$ cells seem to be enriched in early human milk samples and samples from infected mothers, $\mathrm{T}$ cells also seem to increase expression of immune proteins during maternal infection [53].

\section{The PRIMA human milk cohort}

Despite decades of development and improvement of infant formulas, formula fed children are still more susceptible to infections early in life [12]. It is largely unknown what impact the different human milk components have on immune development and how they reduce the susceptibility to infections and allergic diseases. Therefore, we initiated the PRIMA human milk cohort in which we will examine the components in human milk that may protect children from infections, in particular respiratory infections, and allergic diseases.

The added value of this cohort is its strong focus on infectious diseases and allergic disease combined with a detailed immunological analyses of the samples on various components (Additional file 2: supplemental protocol). Added to that, we will collect prospective data with longitudinal breastfeeding sampling in $\mathrm{n}=1000$ children, spread out over multiple winter seasons.

\section{Methods \\ Objectives \\ Primary objective}

To identify components in human milk associated with decreased risk of parent-reported medically attended respiratory tract infections (MARI) during the first year of life in healthy term breastfed infants.

\section{Secondary objectives}

- To identify which components, or combination of components, in human milk are protective against any parent-reported (medically attended) and physician-reported infections during the first year of life.

- To identify which components, or combination of components, in human milk are protective against the development of parent-reported, physicianreported allergic diseases during the first year of life.

- To identify (within a subgroup) which routes are involved in protection transferred from mother to child (human milk, cord blood and amniotic fluid) and are protective against any parent-reported, physician-reported infections/allergic diseases during the first year of life.

- To examine the underlying mechanism of action of the components that have been identified to be protective against MARI, other infections and/or allergic diseases.

\section{Outcomes}

\section{Primary clinical endpoint}

The number of parent-reported medically attended respiratory infections during the first year of life.

\section{Secondary clinical endpoints}

- The number of physician-reported medically attended respiratory infections during the first year of life.

- The number of other parent-reported infections during the first year of life.

- The number of parent-reported allergic diseases (e.g. atopic dermatitis and egg/cow's milk allergies) during the first year of life.

- The number of other physician-reported infections during the first year of life.

- The number of physician-reported allergies during the first year of life.

- The number of antibiotic courses during the first year of life

\section{General study design}

In this prospective, observational cohort we will recruit a total of 1000 mother-child pairs during the first month postpartum. The study population will consist of healthy mothers and children in the Utrecht region, the Netherlands, that already started breastfeeding. Human milk samples will be collected at one week, one month, three months and six months if possible (Fig. 1). These 


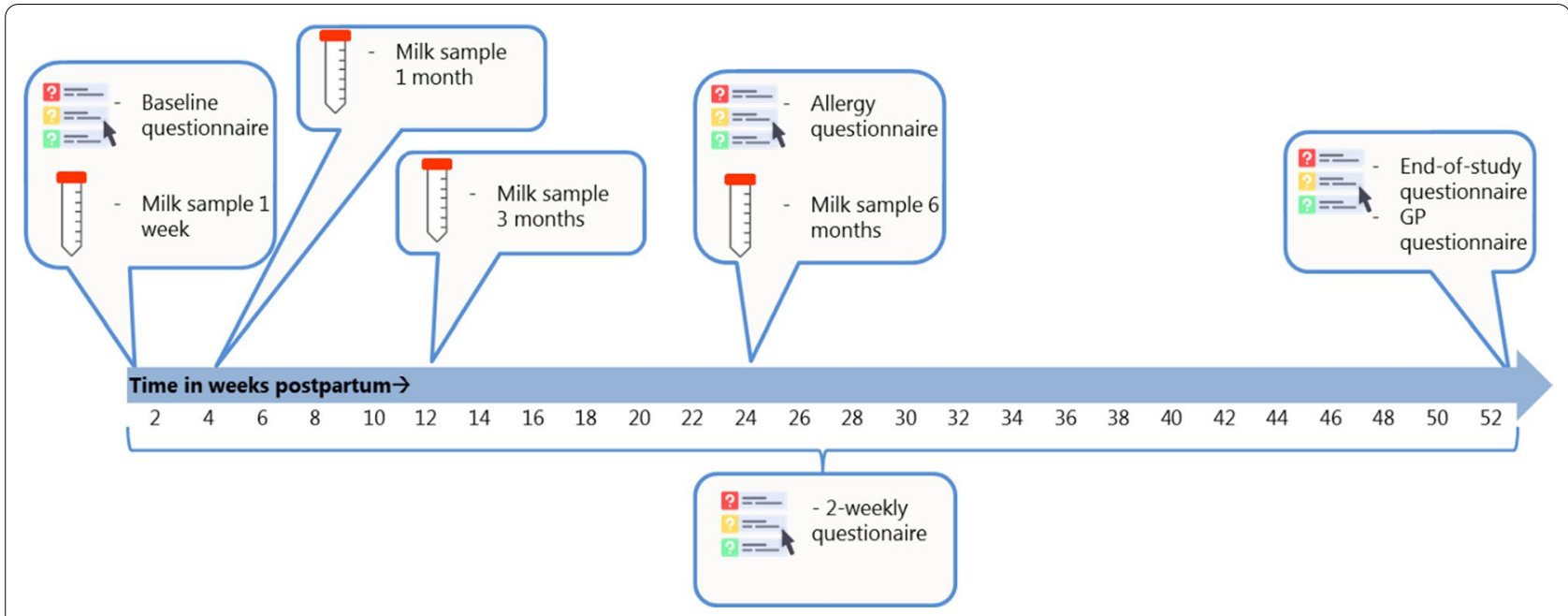

Fig. 1 Overview of sampling and timing of questionnaire: overview of timing sample collection and questionnaires sent to parents

timepoints span colostrum/transitional milk sample (within first week postpartum), early mature (one month sample) and late mature samples (three and six months) $[54,55]$. During the first year of follow up, mothers will report their and their infant's susceptibility to infectious and allergic diseases by completing digital questionnaires sent by email every two weeks until the child reaches one year of age. After the infant has reached the age of one year, their general practitioner will be contacted to collect clinical data on infectious episodes, e.g. antibiotic use and sputum cultures during the first year of life (Additional file 1: Fig S1). In a nested subgroup $(n=20)$, we will collect additional samples of maternal blood, cord blood, amniotic fluid and neonatal saliva and neonatal faecal samples. This subgroup will be used as a pilot to study the effect of all different routes through which mothers may transfer immune active components.

The milk sample composition will be linked to clinical data in order to identify the components or combination of components associated with medically attended respiratory infections, other infections and/or allergies. The human milk samples will be stored in the biobank facility of the UMC Utrecht for future research, securing quality of sample storage. The study recruitment started in March 2019 and is still recruiting participants. The expected end date of recruitment will be in June 2023. Consequently the study will end one year later in June 2024. The study is registered in The Netherlands Trial Register (https://www.trialregister.nl/) under protocol number NL9056.

\section{Study participants}

Participants are recruited at the obstetric wards of the University Medical Centre Utrecht (tertiary centre) and the Diakonessenhuis hospital Utrecht (secondary centre). Participants are recruited in two different ways. Mothers that are admitted to the obstetric wards of either hospitals, will be informed by their midwives about the study. Mothers are asked approval to share their contact details with the researchers of the PRIMA cohort. Mothers that gave consent to share their contact details are contacted consequently.

Mothers that give birth in the primary care birth centre of the University Medical Centre Utrecht will receive a letter from their midwives at discharge. This letter contains information about the study and the possibility to opt out to be contacted by the researchers. $48 \mathrm{~h}$ after discharge, researchers will receive only the contact details of those parents that did not indicate objection to be contacted.

Mothers are screened by eligibility by the researchers during the first phone call. Additional information about the study will be provided and whenever parents are willing to participate, they will be send the patient information folder and the first house visit to collect milk and informed consents are scheduled.

Inclusion is performed within the first week postpartum. An overview of inclusion criteria and study activities overview can be found in Table 1 and Additional file 1: Fig. S1, respectively.

\section{Data collection}

At inclusion, parents will fill out a baseline questionnaire to collect data on (Fig. 1):

- Perinatal and pregnancy health characteristics.

- Family demographics.

- Health status parents. 
Table 1 Eligible criteria for the PRIMA human milk cohort

\begin{tabular}{lll}
\hline & Inclusion criteria & Exclusion criteria \\
\hline Parents & All mother-child pairs that live in a proximity to Utrecht & Acquired or innate immune deficiencies \\
& Parents currently breastfeeding and intending to continue breastfeeding & Insufficient control of the Dutch language \\
until at least 3 months postpartum & Severe cardiac or pulmonary disorders or & other severe organ diseases \\
& & Severe prematurity defined as GA<32 weeks
\end{tabular}

- Vaccination status of the mother. After inclusion, participants will receive a questionnaire every 2 weeks collecting data about:

- Infectious episodes, allergic symptoms, feeding habits, day-care attendance and growth characteristics of infant.

- Doctor visits, diagnoses made and medication prescribed to infant.

- Medication use and health status mother

- Any changes in demographics.

At 6 and 12 months, an additional questionnaire will be added to the 2-weekly questionnaire to record any allergic disease manifestations (Fig. 1). This questionnaire is based on the EuroPrevall infant cohort questionnaires that were designed to investigate the prevalence of allergies in the European population [56]. At twelve months, the vaccination status of the infant, changes in household (e.g. number of children or pets) and more detailed information about any hospital admission of the infant are recorded (Fig. 1).

\section{Sample processing}

Samples are collected by participants at home, either by hand or by breast pump. Within $24 \mathrm{~h}$, study personnel will collect the samples and will note time of expression, method of expression and whether milk was collected before, during or after a feeding or whether it was a whole feeding sample collected. Participants will store the milk samples in their own fridge until study personnel collects and transfers the samples on ice to the UMC Utrecht laboratory. After milk expression, milk is stored on ice or in the fridge until processing. We record what time the sample was expressed and whether it is a whole milk sample or milk collected only before or after a whole feeding. Within 24-h after collection, samples will be processed according to the protocol of Zonneveld et al., using 600 times gravity instead of 3000 times gravity to preserve living cells (Additional file 2: Fig. S2) [57]. After processing, milk supernatant and cream fractions will be stored in cryotubes at $-80{ }^{\circ} \mathrm{C}$. Fresh cell fractions will be used for T cell assays. Antibody and HMO composition is measured in milk supernatant. Samples that will be used for EV analysis, will be processed within 30 min after collection following the above mentioned protocol of Zonneveld et al. [57]. To ensure samples are processed within $30 \mathrm{~min}$, only participants that live in close proximity to the lab and that had sufficient milk production form the first week postpartum are selected for EV isolation. Study personnel is present at moment of milk collection and transfer samples within $20 \mathrm{~min}$ at 37C6 to our laboratory. Total time of transport is recorded for each sample.

\section{Sample analysis}

The milk samples collected in this cohort will be analysed for antibody and oligosaccharide composition (fraction D, Additional file 2: Fig. S2). EV and T cell composition will be analysed in subgroups. The HMO composition is determined by mass spectrometry (MS) based approaches. Recent advances in Liquid chromatography mass spectrometry (LC-MS) enables detection of a vast range of HMOs and to determine relative $\mathrm{HMO}$ concentration [58]. A recently published LC-MS assay has shown to successfully detect semi-quantitively human milk tri-, tetra-, penta- and hexaoses in a single LC-MS assay run[58].

Next to HMOs, samples will be analysed for antibody composition using a bead-based multiplex assay. It has been previously shown that bead-based multiplex assays can reliably measure antibody titres against several pathogens in blood and are an effective means of assessing immunity in a population [59-63]. After optimization, we will use a bead-based multiplex to determine pathogen specific antibodies, e.g. Severe acute respiratory syndrome coronavirus 2 (SARS-CoV-2), Cytomegalovirus (CMV), Bordetella pertussis, pneumococcus, RSV and influenza-specific antibodies [59-62, 64]. Additionally, we will also use a bead-based multiplex assay to determine the concentration of all human antibody isotypes and their subclasses [59-62]. Preliminary data showed that it is also feasible to use these assays in human milk samples, though we will have to optimise these multiplex assays further for the measurement of human milk antibody titres.

In a randomly selected subgroup, we will analyse $\mathrm{T}$ cell profiles and functional skewing with flow cytometry 
as well as their transcriptome and T-cell receptor (TCR) repertoire using single-cell RNA/TCR- sequencing.

\section{Statistical considerations}

We base our sample size calculation on the variation in 2'-FL concentration, as this is one of the most abundant HMOs present in human milk [65]. The sample size of 1000 participants has an $80 \%$ power to detect a ratio of the means of 0.91 in those with vs. those without a parent-reported medically attended respiratory infection using a two-sided two-sample $\mathrm{t}$-test, assuming that $10 \%$ of the cohort participants have a parent-reported medically attended respiratory infection $(\mathrm{N}=100)$ and that the 2'-FL HMO concentration in the population has a mean of $3 \mathrm{~g} / \mathrm{L}$, a standard deviation of $1 \mathrm{~g} / \mathrm{L}$, and a normal distribution [65]. The significance level (alpha) is 0.05. The sample size was calculated using Power Analysis for Sample Size (PASS) 2008.

The primary aim of this study is to identify components in human milk associated with decreased risk of parentreported MARI. To this end we will analyse the association between 2'FL concentration and the MARI during the first year of life using regression analysis. The possible association between 2'-FL concentrations and the number of parent-reported medically attended respiratory infections during the first year of life will be determined using multivariable Poisson regression analysis, adjusting for potential confounders. Potential confounders will be identified by a literature search, complemented by expert knowledge. We will analyze whether the moment of maternal atopy, birth month, duration of lactation postpartum, season, socio-economic status and ethnicity modify the effect of HMOs on the number of respiratory infections.

For our secondary analysis, we will analyse the relationship between HMOs (other than 2'FL) or antibodies and MARI in a similar fashion as 2'FL using regression analysis and Poisson analysis.

For the secondary analysis, the statistical analysis plan will be based on the cohort of 1000 mother child pairs, providing milk samples as well as consecutive questionnaire data related to parent-reported medically attended respiratory infection (MARI). Based on the parent reported respiratory tract infection symptoms, the number infectious episodes during the first year of life will be defined and separates the cohort into two groups (high frequency of infection vs low frequency of infection). We will analyze the relationship between HMOs and antibodies and MARI with a machine-learning based multivariate analysis. We will use recursive ensemble feature selection (REFS) algorithm [66] to reduce the variables to the minimum necessary set to differentiate between the two groups (signature). REFS has been applied successfully to different types of complex biological data and has been shown to have better accuracy [67] than univariate methods and stable enough between datasets [68]. Finally, to validate our results we will use metrics such as area under the curve (AUC) and receiver operating characteristic (ROC) curve $[69,70]$ to validate the diagnostic prediction using the found signature. A similar approach can be followed regarding studying the relation between parent-reported, physician-reported allergic diseases during the first year of life (high frequency of allergy vs low frequency of allergy) and HMOs and antibodies in human milk.

In case there is loss to follow-up because parents stop to report symptoms, we will use the data retrieved from the general practioner to estimate incidence of MARI. If this is not possible, we will impute the missing data using multiple imputation. We will impute missing data using multiple imputation.

\section{Discussion}

Respiratory infections are still a major cause of burden of disease and child death, RSV being the most common found pathogen $[4,17,71]$. In 2015, the worldwide death of 118,200 children under five years of age were attributed to RSV [72]. Protection from respiratory infections will not only lead to a reduction in childhood mortality or other complications, but protection from viral infections is also associated with reduction of other diseases, like recurrent wheeze during first year of life and even asthma [73, 74]. Infants can be protected by vaccinations either given early after birth or by maternal vaccination during pregnancy [75]. These vaccinations elicit a pathogen-specific immune response, offering protection to these specific pathogen only [75]. Currently, no effective (maternal) vaccination is available in the clinic to protect from RSV infections. An important and validated strategy to reduce the number of respiratory infections is breastfeeding [12, 17]. Despite all efforts taken, still, the number of children being exclusively breastfed for six months remains low in some regions. In 2006-2012, only an estimated 25\% of infants in the World Health Organisation (WHO) European Region were exclusively breastfed for the first 6 months, which is far below the WHO recommendation of 6 months exclusive followed partial breastfeeding until the age of two.

Understanding the composition of breast milk in relation to disease susceptibility in early life will guide the development of much needed interventions for infants that cannot be exclusively breast fed. At the present moment, there is a lack in knowledge about the specific components or combination of components present in human milk that support the protective capacity against infections. In order to be able to improve safety and 
efficacy of infant formulas, it first has to be established which components including their underlying mechanism of action in breastfeeding are essential to protect against infections.

The PRIMA human milk cohort is designed to study human milk components in a novel public-private collaboration. The PRIMA human milk cohort will be one of the largest cohorts combining both longitudinal human milk samples with prospective clinical data collection. This initiative will offer a unique possibility to add scientific understanding regarding the protective effect and influence of specific human milk derived components on the protection against respiratory infections and allergies early in life. Data and samples from this cohort will be used to study the underlying mechanism of several components in human milk which might lead to the development of new concepts/hypotheses for the management and therapy of immune related disorders such as allergies and infections.

\begin{abstract}
Abbreviations
EV: Extracellular vesicle; RSV: Respiratory syncytial virus; IFV: Influenza virus; PIV: Parainfluenza; MPV: Human metapneumovirus; Cl: Confidence interval; HMO(S): Human milk oligosaccharide(s); IgA: Immunoglobulin A; IgG: Immunoglobulin G; 2'FL: 2'-Fucosyllactose; MARI: Medically attended respiratory infection; MS: Mass spectrometry; LC-MS: Liquid chromatography mass spectrometry; SARS-CoV-2: Severe acute respiratory syndrome coronavirus 2; CMV: Cytomegalovirus; TCR: T-cell receptor; PASS: Power analysis for sample size; WHO: World Health Organisation; GA: Gestational age.
\end{abstract}

\section{Supplementary Information}

The online version contains supplementary material available at https://doi. org/10.1186/s12879-022-07107-w.

Additional file 1: Figure S1. Study activities overview: overview of all study activities. Figure S2. Sample processing in PRIMA human milk cohort: A) Human milk samples are centrifuged for 10 min at $600 \mathrm{~g}$ in order to remove the cream (layer on top, indicated in yellow) and cells (collected in pellet on the bottom, indicated in dark grey). B) Milk supernatant is collected by pipetting through cream layer without disturbing the cell pellet and subsequently transferred into a new tube. C) Collected $600 \mathrm{~g}$ supernatant is again centrifuged for another $10 \mathrm{~min}$ at $600 \mathrm{~g}$. D) Milk supernatant is collected by pipetting through the residual cream and transferred to and aliquoted into clean cryotubes, and stored at $-80^{\circ} \mathrm{C}$ until further analysis. E) The cream layer is removed without disturbing the cellular pellet and transferred into cryotubes stored at $-80^{\circ} \mathrm{C}$ for further analysis. F) $20 \mathrm{~mL}$ PBS is added to rinse and cells are spun at $600 \mathrm{~g}$ for $10 \mathrm{~min}$, after which the supernatant is discarded and cells are kept on ice until further processing for analysis G). Milk EVs are processed further according to the Zonneveld protocol at $3000 \mathrm{~g}$.

Additional file 2. PRIMA research protocol METC Utrecht version 4A: this file contains the protocol including the novel additions for collection of dietary information from mother.

\section{Acknowledgements}

The following respondents of the PRIMA Initiative Group opted to have their name acknowledged: André C. Knulst; Department of Dermatology/Allergology, University Medical Center Utrecht, Utrecht, The Netherlands. C. Kors van der Ent; Department of Pediatric Pulmonology and Allergology, Wilhelmina Children's Hospital/University Medical Center, Utrecht University, Utrecht, the
Netherlands. Debbie van Baarle; Center for Translational Immunology, University Medical Center Utrecht, Utrecht, Netherlands. Centre for Infectious Disease Control, National Institute for Public Health and the Environment (RIVM), Bilthoven, The Netherlands. Marca HM Wauben, Department of Biomolecular Health Sciences, Faculty of Veterinary Medicine, Utrecht University, Utrecht, the Netherlands. Nynke Y Rots, Center for Translational Immunology, University Medical Center Utrecht, Utrecht, Netherlands. Centre for Infectious Disease Control, National Institute for Public Health and the Environment (RIVM), Bilthoven, The Netherlands. Elisabeth AM Sanders; Center for Translational Immunology, University Medical Center Utrecht, Utrecht, Netherlands. Centre for Infectious Disease Control, National Institute for Public Health and the Environment (RIVM), Bilthoven, The Netherlands. Manon JNL Benders; Department of Neonatology, Wilhelmina Children's Hospital/University Medical Centre Utrecht, Utrecht, The Netherlands.Saskia A Overbeek; Global Centre of Excellence Immunology, Danone Nutricia Research B.V., Utrecht, Netherlands. Laura AMP Meulenbroek; Global Centre of Excellence Immunology, Danone Nutricia Research B.V., Utrecht, Netherlands. Bernd Stahl; Division of Pharmacology, Faculty of Science, Utrecht Institute for Pharmaceutical Sciences, Utrecht University, Utrecht, Netherlands. Global Centre of Excellence Immunology, Danone Nutricia Research B.V., Utrecht, Netherlands. Global Centre of Excellence Human Milk Research and Analytical Sciences, Danone Nutricia Research B.V., Utrecht, Netherlands. Aletta D Kraneveld; Division of Pharmacology, Faculty of Science, Utrecht Institute for Pharmaceutical Sciences, Utrecht University, Utrecht, Netherlands. Brigitte J. M. Buiteman; Department of Paediatric Immunology and Infectious Diseases, Wilhelmina Children's Hospital/University Medical Center Utrecht, Utrecht, Netherlands. Tanja Voogt; Department of Obstetrics and Gynaecology, Diakonessenhuis, Utrecht, the Netherlands. Barbara van der Meij; Department of Obstetrics and Gynaecology, Diakonessenhuis, Utrecht, the Netherlands. Butsabong Lerkvaleekul; Center for Translational Immunology, University Medical Center Utrecht, Utrecht, Netherlands. Eline Voogd, Global Centre of Excellence Human Milk Research and Analytical Sciences, Danone Nutricia Research B.V., Utrecht, Netherlands. Christianne S. Lammers; Department of Paediatric Immunology and Infectious Diseases, Wilhelmina Children's Hospital/University Medical Center Utrecht, Utrecht, Netherlands. Tariq A. Lalmahomed; Center for Translational Immunology, University Medical Center Utrecht, Utrecht, Netherlands. Iris M. Brus; Center for Translational Immunology, University Medical Center Utrecht, Utrecht, Netherlands. Daphne M. M. van Meerwijk; Center for Translational Immunology, University Medical Center Utrecht, Utrecht, Netherlands. Sophie I. E. Jepma; Center for Translational Immunology, University Medical Center Utrecht, Utrecht, Netherlands.

\section{Authors' contributions}

AvS, KOR and LB wrote the manuscript. LB, JL and BvL supervised and initiated the project. KB and WdW supported resources for recruitment of study participants. AvS, SP, TL, FvW, MN, AH, CL, GdH, MvH, JG, LK, LV, CdT, AL, JL, BvL and LB were involved in setting up the methodology of the trial. AvS and MN performed validation experiments. All authors were involved in the revision of the manuscript. All authors read and approved the final manuscript.

\section{Funding}

This research was supported by Regio Deal Foodvalley (grant nr 162135), the WKZ research fund-Nutricia call 2020 "Breast milk T cells: frontline protection for infants and mothers?" and from Danone Nutricia Research BV as part of a UMC Utrecht-Danone Nutricia Research BV collaboration grant "Early Life Nutrition and Immune Development" (Ministerie van Volksgezondheid, Welzijn en Sport, Universitair Medisch Centrum Utrecht, Danone Nutricia Research BV). The funders had no role in the study design, in the collection, analysis and interpretation of data. The funders had no role in the final decisions on the interpretation and dissemination of the results.

\section{Availability of data and materials}

As the current manuscript describes the study protocol and no other data, we do not have any raw data to share at the moment.

\section{Declarations}

\section{Ethics approval and consent to participate}

The PRIMA cohort has been approved by the Medical Research Ethics Committee of the UMC Utrecht, the Netherlands (reference number: SL/ 
avd/20/500151, protocol ID: 20-578/X-D). All participating parents gave written consent to participate in this cohort.

\section{Consent for publication}

Not applicable.

\section{Competing interests}

The study was funded by grants from the Dutch Ministry of Health, Welfare and Sport, the Wilhelmina Children's Hospital and Nutricia B.V. None of the authors have a competing financial interest in relation to the presented work; JG, LK and BL are (partly) employed by Danone Nutricia Research.

\section{Author details}

${ }^{1}$ Center for Translational Immunology, University Medical Center Utrecht, Utrecht, The Netherlands. ${ }^{2}$ Department of Biostatistics and Research Support, Clinical Trial Methodology, Julius Center for Health Sciences and Primary Care, University Medical Center Utrecht, Utrecht, The Netherlands. ${ }^{3}$ Department of Gynaecology and Obstetrics, University Medical Center Utrecht, Utrecht, The Netherlands. ${ }^{4}$ Department of Pediatrics, Diakonessenhuis, Utrecht, The Netherlands. ${ }^{5}$ Department of Pediatric Pulmonology and Allergology, Wilhelmina Children's Hospital/University Medical Center, Utrecht University, Utrecht, The Netherlands. ${ }^{6}$ Department of Dermatology/Allergology, University Medical Center Utrecht, University of Utrecht, Utrecht, The Netherlands. ${ }^{7}$ Centre for Infectious Disease Control, National Institute for Public Health and the Environment (RIVM), Bilthoven, The Netherlands. ${ }^{8}$ Department of Biomolecular Health Sciences, Faculty of Veterinary Medicine, Utrecht University, Utrecht, The Netherlands. ${ }^{9}$ Division of Pharmacology, Utrecht Institute for Pharmaceutical Sciences, Utrecht University, Utrecht, The Netherlands. ${ }^{10}$ Danone Nutricia Research, Utrecht, The Netherlands. ${ }^{11}$ Department of Paediatric Immunology and Infectious Diseases, Wilhelmina Children's Hospital/ University Medical Center Utrecht, Utrecht, The Netherlands. ${ }^{12}$ Department of Neonatology, Wilhelmina Children's Hospital, University Medical Centre Utrecht, Utrecht, The Netherlands. ${ }^{13}$ ReSViNET Foundation, Zeist, The Netherlands.

Received: 22 December 2020 Accepted: 29 January 2022

Published online: 14 February 2022

\section{References}

1. Kutsaya A, Teros-Jaakkola T, Kakkola L, Toivonen L, Peltola V, Waris M, et al. Prospective clinical and serological follow-up in early childhood reveals a high rate of subclinical RSV infection and a relatively high reinfection rate within the first 3 years of life. Epidemiol Infect. 2016;144(8):1622-33.

2. Wang $\mathrm{H}$, Naghavi M, Allen C, Barber RM, Bhutta ZA, Carter A, et al. Global, regional, and national life expectancy, all-cause mortality, and cause-specific mortality for 249 causes of death, 1980-2015: a systematic analysis for the Global Burden of Disease Study 2015. Lancet. 2016;388(10053):1459-544.

3. Pneumonia Etiology Research for Child Health Study G. Causes of severe pneumonia requiring hospital admission in children without HIV infection from Africa and Asia: the PERCH multi-country case-control study. Lancet. 2019;394(10200):757-79.

4. Nair H, Nokes DJ, Gessner BD, Dherani M, Madhi SA, Singleton RJ, et al. Global burden of acute lower respiratory infections due to respiratory syncytial virus in young children: a systematic review and meta-analysis. Lancet. 2010;375(9725):1545-55.

5. Reeves RM, van Wijhe M, Tong S, Lehtonen T, Stona L, Teirlinck AC, et al. Respiratory syncytial virus-associated hospital admissions in children younger than 5 years in 7 European countries using routinely collected datasets. J Infect Dis. 2020;222(Supplement_7):S599-605.

6. Yu ZT, Chen C, Newburg DS. Utilization of major fucosylated and sialylated human milk oligosaccharides by isolated human gut microbes. Glycobiology. 2013;23(11):1281-92.

7. Isaacs D. Should respiratory care in preterm infants include prophylaxis against respiratory syncytial virus? The case against. Paediatr Respir Rev. 2013;14(2):128-9.

8. Meijboom MJ, Rozenbaum MH, Benedictus A, Luytjes W, Kneyber MC, Wilschut JC, et al. Cost-effectiveness of potential infant vaccination against respiratory syncytial virus infection in The Netherlands. Vaccine. 2012;30(31):4691-700.

9. Bos JM, Rietveld E, Moll HA, Steyerberg EW, Luytjes W, Wilschut JC, et al. The use of health economics to guide drug development decisions: determining optimal values for an RSV-vaccine in a model-based scenario-analytic approach. Vaccine. 2007;25(39-40):6922-9.

10. Jansen AG, Sanders EA, Hoes AW, van Loon AM, Hak E. Influenza- and respiratory syncytial virus-associated mortality and hospitalisations. Eur Respir J. 2007;30(6):1158-66.

11. Fauroux B, Simões EAF, Checchia PA, Paes B, Figueras-Aloy J, Manzoni P, et al. The burden and long-term respiratory morbidity associated with respiratory syncytial virus infection in early childhood. Infect Dis Therapy. 2017;6(2):173-97.

12. Lawrence RM, Lawrence RA. Breastfeeding: more than just good nutrition. Pediatr Rev. 2011;32(7):267-80.

13. Munblit D, Peroni DG, Boix-Amorós A, Hsu PS, Van't Land B, Gay MCL, et al. Human milk and allergic diseases: an unsolved puzzle. Nutrients. 2017. https://doi.org/10.3390/nu9080894.

14. Ajetunmobi OM, Whyte B, Chalmers J, Tappin DM, Wolfson L, Fleming $M$, et al. Breastfeeding is associated with reduced childhood hospitalization: evidence from a Scottish Birth Cohort (1997-2009). J Pediatr. 2015;166(3):620-625 e4.

15. Walker WA, lyengar RS. Breast milk, microbiota, and intestinal immune homeostasis. Pediatr Res. 2015;77(1-2):220-8.

16. Duijts L, Jaddoe VW, Hofman A, Moll HA. Prolonged and exclusive breastfeeding reduces the risk of infectious diseases in infancy. Pediatrics. 2010;126(1):e18-25

17. Shi T, Balsells E, Wastnedge E, Singleton R, Rasmussen ZA, Zar HJ, et al. Risk factors for respiratory syncytial virus associated with acute lower respiratory infection in children under five years: systematic review and meta-analysis. J Glob Health. 2015;5(2): 020416.

18. Rietveld E, De Jonge HC, Polder JJ, Vergouwe Y, Veeze HJ, Moll HA, et al. Anticipated costs of hospitalization for respiratory syncytial virus infection in young children at risk. Pediatr Infect Dis J. 2004;23(6):523-9.

19. Levine OS, Farley M, Harrison LH, Lefkowitz L, McGeer A, Schwartz B. Risk factors for invasive pneumococcal disease in children: a populationbased case-control study in North America. Pediatrics. 1999;103(3):E28.

20. Dharan NJ, Sokolow LZ, Cheng PY, Gargiullo P, Gershman K, Lynfield R, et al. Child, household, and caregiver characteristics associated with hospitalization for influenza among children 6-59 months of age: an emerging infections program study. Pediatr Infect Dis J. 2014;33(6):e141-50.

21. Lamberti LM, Fischer Walker CL, Noiman A, Victora C, Black RE. Breastfeeding and the risk for diarrhea morbidity and mortality. BMC Public Health. 2011;11(Suppl 3):S15.

22. Lodge CJ, Tan DJ, Lau MX, Dai X, Tham R, Lowe AJ, et al. Breastfeeding and asthma and allergies: a systematic review and meta-analysis. Acta Paediatr. 2015;104(467):38-53.

23. Klopp A, Vehling L, Becker AB, Subbarao P, Mandhane PJ, Turvey SE, et al. Modes of infant feeding and the risk of childhood asthma: a prospective birth cohort study. J Pediatr. 2017;190:192-292.

24. Azad MB, Vehling L, Lu Z, Dai D, Subbarao P, Becker AB, et al. Breastfeeding, maternal asthma and wheezing in the first year of life: a longitudinal birth cohort study. Eur Respir J. 2017. https://doi.org/10.1183/13993003. 02019-2016.

25. Flohr C, Nagel G, Weinmayr G, Kleiner A, Strachan DP, Williams HC, et al. Lack of evidence for a protective effect of prolonged breastfeeding on childhood eczema: lessons from the International Study of Asthma and Allergies in Childhood (ISAAC) Phase Two. Br J Dermatol. 2011;165(6):1280-9.

26. Pesonen M, Kallio MJ, Ranki A, Siimes MA. Prolonged exclusive breastfeeding is associated with increased atopic dermatitis: a prospective follow-up study of unselected healthy newborns from birth to age 20 years. Clin Exp Allergy. 2006;36(8):1011-8.

27. Giwercman C, Halkjaer LB, Jensen SM, Bonnelykke K, Lauritzen L, Bisgaard $H$. Increased risk of eczema but reduced risk of early wheezy disorder from exclusive breast-feeding in high-risk infants. J Allergy Clin Immunol. 2010;125(4):866-71.

28. Han SM, Binia A, Godfrey KM, El-Heis S, Cutfield WS. Do human milk oligosaccharides protect against infant atopic disorders and food allergy? Nutrients. 2020. https://doi.org/10.3390/nu12103212. 
29. Lodge CJ, Lowe AJ, Milanzi E, Bowatte G, Abramson MJ, Tsimiklis H, et al. Human milk oligosaccharide profiles and allergic disease up to 18 years. J Allergy Clin Immunol. 2020. https://doi.org/10.1016/j.jaci.2020.06.027.

30. Mazur NI, Martinón-Torres F, Baraldi E, Fauroux B, Greenough A, Heikkinen $T$, et al. Lower respiratory tract infection caused by respiratory syncytial virus: current management and new therapeutics. Lancet Respir Med. 2015;3(11):888-900.

31. Breakey AA, Hinde K, Valeggia CR, Sinofsky A, Ellison PT. Illness in breastfeeding infants relates to concentration of lactoferrin and secretory Immunoglobulin A in mother's milk. Evol Med Public Health. 2015;2015(1):21-31.

32. Ramani S, Stewart CJ, Laucirica DR, Ajami NJ, Robertson B, Autran CA, et al. Human milk oligosaccharides, milk microbiome and infant gut microbiome modulate neonatal rotavirus infection. Nat Commun. 2018;9(1):5010.

33. Barton SJ, Murray R, Lillycrop KA, Inskip HM, Harvey NC, Cooper C, et al. FUT2 genetic variants and reported respiratory and gastrointestinal illnesses during infancy. J Infect Dis. 2019;219(5):836-43.

34. Arslanoglu S, Moro GE, Boehm G. Early supplementation of prebiotic oligosaccharides protects formula-fed infants against infections during the first 6 months of life. J Nutr. 2007;137(11):2420-4.

35. Moro G, Arslanoglu S, Stahl B, Jelinek J, Wahn U, Boehm G. A mixture of prebiotic oligosaccharides reduces the incidence of atopic dermatitis during the first six months of age. Arch Dis Child. 2006:91(10):814-9.

36. Gruber C, van Stuijvenberg M, Mosca F, Moro G, Chirico G, Braegger CP, et al. Reduced occurrence of early atopic dermatitis because of immunoactive prebiotics among low-atopy-risk infants. J Allergy Clin Immunol. 2010;126(4):791-7.

37. Ranucci G, Buccigrossi V, Borgia E, Piacentini D, Visentin F, Cantarutti L, et al. Galacto-oligosaccharide/polidextrose enriched formula protects against respiratory infections in infants at high risk of atopy: a randomized clinical trial. Nutrients. 2018. https://doi.org/10.3390/nu10030286.

38. Arslanoglu S, Moro GE, Boehm G, Wienz F, Stahl B, Bertino E. Early neutral prebiotic oligosaccharide supplementation reduces the incidence of some allergic manifestations in the first 5 years of life. J Biol Regul Homeost Agents. 2012;26(3 Suppl):49-59.

39. Ayechu-Muruzabal V, van Stigt AH, Mank M, Willemsen LEM, Stahl B, Garssen J, et al. Diversity of human milk oligosaccharides and effects on early life immune development. Front Pediatr. 2018;6:239.

40. Laucirica DR, Triantis V, Schoemaker R, Estes MK, Ramani S. Milk oligosaccharides inhibit human rotavirus infectivity in MA104 cells. J Nutr. 2017;147(9):1709-14.

41. van Erp EA, Lakerveld AJ, de Graaf E, Larsen MD, Schepp RM, Hipgrave Ederveen AL, et al. Natural killer cell activation by respiratory syncytial virus-specific antibodies is decreased in infants with severe respiratory infections and correlates with Fc-glycosylation. Clin Transl Immunol. 2020;9(2): e1112.

42. Zehra S, Khambati I, Vierhout M, Mian MF, Buck R, Forsythe P. Human milk oligosaccharides attenuate antigen-antibody complex induced chemokine release from human intestinal epithelial cell lines. J Food Sci. 2018;83(2):499-508

43. Xiao L, van De Worp WR, Stassen R, van Maastrigt C, Kettelarij N, Stahl $B$, et al. Human milk oligosaccharides promote immune tolerance via direct interactions with human dendritic cells. Eur J Immunol. 2019;49(7):1001-14.

44. Xiao L, Van't Land B, Engen PA, Naqib A, Green SJ, Nato A, et al. Human milk oligosaccharides protect against the development of autoimmune diabetes in NOD-mice. Sci Rep. 2018;8(1):3829.

45. Backhed F, Roswall J, Peng Y, Feng Q, Jia H, Kovatcheva-Datchary P, et al. Dynamics and stabilization of the human gut microbiome during the first year of life. Cell Host Microbe. 2015;17(5):690-703.

46. Rautava S. Early microbial contact, the breast milk microbiome and child health. J Dev Orig Health Dis. 2016;7(1):5-14.

47. Hassiotou F, Hepworth AR, Metzger P, Tat Lai C, Trengove N, Hartmann PE, et al. Maternal and infant infections stimulate a rapid leukocyte response in breastmilk. Clin Transl Immunol. 2013;2(4): e3.

48. Timby N, Domellöf M, Lönnerdal B, Hernell O. Supplementation of infant formula with bovine milk fat globule membranes. Adv Nutr. 2017;8(2):351-5.
49. Verhasselt V, Milcent V, Cazareth J, Kanda A, Fleury S, Dombrowicz D, et al. Breast milk-mediated transfer of an antigen induces tolerance and protection from allergic asthma. Nat Med. 2008;14(2):170-5.

50. Dawod B, Marshall JS. Cytokines and soluble receptors in breast milk as enhancers of oral tolerance development. Front Immunol. 2019;10:16.

51. van den Elsen LWJ, Garssen J, Burcelin R, Verhasselt V. Shaping the gut microbiota by breastfeeding: the gateway to allergy prevention? Front Pediatr. 2019;7:47.

52. Kosaka $\mathrm{N}$, Izumi $\mathrm{H}$, Sekine $\mathrm{K}$, Ochiya T. microRNA as a new immune-regulatory agent in breast milk. Silence. 2010;1(1):7.

53. Twigger AJ, Kuffer GK, Geddes DT, Filgueria L. Expression of granulisyn, perforin and granzymes in human milk over lactation and in the case of maternal infection. Nutrients. 2018. https://doi.org/10.3390/nu10091230.

54. Koletzko B. Human milk lipids. Ann Nutr Metab. 2016;69(Suppl 2):28-40.

55. Ballard O, Morrow AL. Human milk composition: nutrients and bioactive factors. Pediatr Clin North Am. 2013;60(1):49-74.

56. Kummeling I, Mills EN, Clausen M, Dubakiene R, Pérez CF, FernándezRivas $\mathrm{M}$, et al. The EuroPrevall surveys on the prevalence of food allergies in children and adults: background and study methodology. Allergy. 2009:64(10):1493-7.

57. Zonneveld MI, Brisson AR, van Herwijnen MJ, Tan S, van de Lest CH, Redegeld FA, et al. Recovery of extracellular vesicles from human breast milk is influenced by sample collection and vesicle isolation procedures. J Extracell Vesicles. 2014. https://doi.org/10.3402/jev.v3.24215.

58. Mank M, Welsch P, Heck AJR, Stahl B. Label-free targeted LC-ESI-MS(2) analysis of human milk oligosaccharides (HMOS) and related human milk groups with enhanced structural selectivity. Anal Bioanal Chem. 2019;411(1):231-50.

59. van Gageldonk PG, van Schaijk FG, van der Klis FR, Berbers GA. Development and validation of a multiplex immunoassay for the simultaneous determination of serum antibodies to Bordetella pertussis, diphtheria and tetanus. J Immunol Methods. 2008;335(1-2):79-89.

60. de Voer RM, van der Klis FR, Engels CW, Rijkers GT, Sanders EA, Berbers GA. Development of a fluorescent-bead-based multiplex immunoassay to determine immunoglobulin $\mathrm{G}$ subclass responses to Neisseria meningitidis serogroup A and C polysaccharides. Clin Vaccine Immunol. 2008;15(8):1188-93.

61. Smits GP, van Gageldonk PG, Schouls LM, van der Klis FR, Berbers GA. Development of a bead-based multiplex immunoassay for simultaneous quantitative detection of IgG serum antibodies against measles, mumps, rubella, and varicella-zoster virus. Clin Vaccine Immunol. 2012:19(3):396-400

62. Tcherniaeva I, den Hartog G, Berbers G, van der Klis F. The development of a bead-based multiplex immunoassay for the detection of $\lg G$ antibodies to CMV and EBV. J Immunol Methods. 2018;462:1-8.

63. den Hartog $G$, van Binnendijk R, Buisman AM, Berbers GAM, van der Klis FRM. Immune surveillance for vaccine-preventable diseases. Expert Rev Vaccines. 2020;19(4):327-39.

64. den Hartog G, Schepp RM, Kuijer M, GeurtsvanKessel C, van Beek J, Rots $\mathrm{N}$, et al. SARS-CoV-2-specific antibody detection for seroepidemiology: a multiplex analysis approach accounting for accurate seroprevalence. J Infect Dis. 2020;222(9):1452-61.

65. Donovan SM, Comstock SS. Human milk oligosaccharides influence neonatal mucosal and systemic immunity. Ann Nutr Metab. 2016;69(Suppl 2):42-51.

66. Lopez-Rincon A, Mendoza-Maldonado L, Martinez-Archundia M, Schonhuth A, Kraneveld AD, Garssen J, et al. Machine learning-based ensemble recursive feature selection of circulating miRNAs for cancer tumor classification. Cancers (Basel). 2020. https://doi.org/10.3390/cancers12071785.

67. Lopez-Rincon A, Martinez-Archundia M, Martinez-Ruiz GU, Schoenhuth A, Tonda A. Automatic discovery of 100-miRNA signature for cancer classification using ensemble feature selection. BMC Bioinformatics. 2019;20(1):480

68. Metselaar PI, Mendoza-Maldonado L, Li Yim AYF, Abarkan I, Henneman P, Te Velde AA, et al. Recursive ensemble feature selection provides a robust mRNA expression signature for myalgic encephalomyelitis/chronic fatigue syndrome. Sci Rep. 2021;11(1):4541.

69. Simundic AM. Measures of diagnostic accuracy: basic definitions. EJIFCC. 2009;19(4):203-11.

70. Mandrekar JN. Receiver operating characteristic curve in diagnostic test assessment. J Thorac Oncol. 2010:5(9):1315-6. 
71. Williams BG, Gouws E, Boschi-Pinto C, Bryce J, Dye C. Estimates of worldwide distribution of child deaths from acute respiratory infections. Lancet Infect Dis. 2002;2(1):25-32.

72. Shi T, McAllister DA, O'Brien KL, Simoes EAF, Madhi SA, Gessner BD, et al. Global, regional, and national disease burden estimates of acute lower respiratory infections due to respiratory syncytial virus in young children in 2015: a systematic review and modelling study. Lancet. 2017;390(10098):946-58.

73. Blanken MO, Rovers MM, Molenaar JM, Winkler-Seinstra PL, Meijer A Kimpen $\mathrm{J}$, et al. Respiratory syncytial virus and recurrent wheeze in healthy preterm infants. N Engl J Med. 2013;368(19):1791-9.

74. Mejias A, Wu B, Tandon N, Chow W, Varma R, Franco E, et al. Risk of childhood wheeze and asthma after respiratory syncytial virus infection in full-term infants. Pediatr Allergy Immunol. 2020;31 (1):47-56.

75. Marchant A, Sadarangani M, Garand M, Dauby N, Verhasselt V, Pereira L, et al. Maternal immunisation: collaborating with mother nature. Lancet Infect Dis. 2017;17(7):e197-208.

\section{Publisher's Note}

Springer Nature remains neutral with regard to jurisdictional claims in published maps and institutional affiliations.

- fast, convenient online submission

- thorough peer review by experienced researchers in your field

- rapid publication on acceptance

- support for research data, including large and complex data types

- gold Open Access which fosters wider collaboration and increased citations

- maximum visibility for your research: over $100 \mathrm{M}$ website views per year

At BMC, research is always in progress.

Learn more biomedcentral.com/submissions 